\title{
How to construct the discrete symmetries of partial differential equations
}

\author{
P. E. HYDON \\ Department of Mathematics and Statistics, University of Surrey, \\ Guildford GU2 5XH, UK \\ (email: P.Hydon@surrey.ac.uk)
}

(Received in revised form 12 May 2000)

This paper introduces an algorithm for calculating all discrete point symmetries of a given partial differential equation with a known nontrivial group of Lie point symmetries. The method enables the user to determine the discrete symmetries with little more effort than is used to find the Lie symmetries. It is used to obtain the discrete point symmetries of Burgers' equation, the spherical Burgers' equation, and the Harry-Dym equation. The method can be extended to some types of nonlocal symmetry; we derive the quasi-local discrete symmetries of a system of PDEs from gas dynamics.

\section{Introduction}

Discrete symmetries of Partial Differential Equations (PDEs) are important for various reasons. For instance, to understand how a system changes its stability, one must first know its discrete and continuous symmetries, in order to apply equivariant bifurcation theory correctly. Discrete symmetries involving charge conjugation, parity change, and time-reversal play a key rôle in quantum field theories. Nonlocal discrete symmetries such as auto-Bäcklund transformations are important in the study of integrable systems. Discrete symmetries are also used to simplify the numerical computation of solutions of PDEs, and to create new exact solutions from known solutions.

Continuous groups of symmetries can be constructed systematically using Lie's method, which linearizes the determining equations that are satisfied by all symmetries $[1,6,8,10]$. However, for discrete symmetries, no such linearization is possible. The problem of solving the determining equations directly is usually intractable; until now, the only other means of obtaining discrete symmetries has been to use an ansatz (see Gaeta \& Rodríguez [2]). However, this approach can only yield symmetries in the class admitted by the ansatz; a given system may have other discrete symmetries outside that class.

This paper introduces a constructive technique that uses the Lie point symmetries of a given system of PDEs to determine all of the discrete point symmetries. The method is easy to use, and can be applied to any system whose Lie point symmetries are known (and non-trivial). Most PDEs that arise from mathematical models are in this category.

The technique is based on the observation that every point symmetry yields an automorphism of the Lie algebra of Lie point symmetry generators. This results in a set of auxiliary equations that are satisfied by all point symmetries; these equations are solvable 
by standard techniques. If the Lie algebra is non-abelian, the auxiliary equations may be considerably simplified before they are solved. Typically, the solution contains unknown constants or functions of integration. Substituting the solution into the original (nonlinear) determining equations, and then factoring out the known continuous symmetries, produces a list of all discrete point symmetries. The method readily extends to other types of symmetries, as is described elsewhere [4, 5].

\section{The determining equations}

Consider a given regular system of PDEs,

$$
\Delta(x, u)=0,
$$

that involves $M$ dependent variables, $u=\left\{u^{1}, \ldots u^{M}\right\}$, and $N$ independent variables, $x=\left\{x^{1}, \ldots, x^{N}\right\}$. A diffeomorphism

$$
\Gamma:(x, u) \mapsto(\hat{x}(x, u), \hat{u}(x, u))
$$

is a point symmetry of the given system if $\Gamma$ maps the set of solutions to itself. This happens if

$$
\Delta(\hat{x}, \hat{u})=0 \quad \text { when } \quad \Delta(x, u)=0 .
$$

The symmetry condition (2.3) can be split into a system of determining equations for $(\hat{x}, \hat{u})$. Typically, the determining equations are nonlinear and highly-coupled, and they cannot be solved by a direct approach. (Even for Ordinary Differential Equations (ODEs), the determining equations are almost always too difficult to solve. I know of only one instance in which these equations have been solved directly [9]; the authors used computer algebra to produce a differential Gröbner basis for the determining equations of a second-order ODE.)

Although the discrete symmetries of a given PDE cannot usually be obtained directly, the Lie point symmetries are generally easy to find. They are of the form

$$
\begin{array}{cc}
\hat{x}^{s}=x^{s}+\epsilon \xi^{s}(x, u)+O\left(\epsilon^{2}\right), & s=1, \ldots, N, \\
\hat{u}^{\sigma}=u^{\sigma}+\epsilon \eta^{\sigma}(x, u)+O\left(\epsilon^{2}\right), & \sigma=1, \ldots, M,
\end{array}
$$

and they are obtained by linearizing the symmetry condition about $\epsilon=0$ (see elsewhere $[1,6,8,10]$ for further details). Routines for calculating Lie point symmetries are available for use with all major computer algebra packages (see Hereman [3]). Henceforth, we shall assume that the Lie point symmetries have been found. For simplicity, we also assume that the set of infinitesimal generators forms an $R$-dimensional Lie algebra, $\mathscr{L}$, which has a basis

$$
X_{i}=\xi_{i}^{s}(x, u) \partial_{x^{s}}+\eta_{i}^{\sigma}(x, u) \partial_{u^{\sigma}}, \quad i=1, \ldots, R .
$$

(The usual summation convention applies.) It is worthwhile choosing a basis in which the commutator relations

$$
\left[X_{i}, X_{j}\right]=c_{i j}^{k} X_{k}
$$

are as simple as possible. 
The one-parameter group of continuous symmetries generated by $X_{i}$ is

$$
\Gamma_{i}(\epsilon)=\exp \left(\epsilon X_{i}\right)
$$

Now suppose that

$$
\Gamma:(x, u) \mapsto(\hat{x}, \hat{u})
$$

is any symmetry of the given PDE. Then

$$
\hat{\Gamma}_{i}(\epsilon)=\Gamma \Gamma_{i}(\epsilon) \Gamma^{-1}=\exp \left(\epsilon \Gamma X_{i} \Gamma^{-1}\right),
$$

is a one-parameter group of continous symmetries, which is generated by

$$
\hat{X}_{i}=\Gamma X_{i} \Gamma^{-1} \text {. }
$$

In particular,

$$
\hat{X}_{i} \hat{x}^{s}=\Gamma X_{i} x^{s}=\Gamma \xi_{i}^{s}(x, u)=\xi_{i}^{s}(\hat{x}, \hat{u}),
$$

and similarly,

$$
\hat{X}_{i} \hat{u}^{\sigma}=\eta_{i}^{\sigma}(\hat{x}, \hat{u})
$$

Therefore

$$
\hat{X}_{i}=\xi_{i}^{s}(\hat{x}, \hat{u}) \partial_{\hat{x}^{s}}+\eta_{i}^{\sigma}(\hat{x}, \hat{u}) \partial_{\hat{u}^{\sigma}},
$$

and so each $\hat{X}_{i}$ has exactly the same functional form as the corresponding $X_{i}$, but with $(\hat{x}, \hat{u})$ replacing $(x, u)$ in $(2.4)$. Thus the transformed generators $\hat{X}_{i}, i=1, \ldots, R$ constitute a basis for $\mathscr{L}$, with exactly the same commutator relations as the original basis:

$$
\left[\hat{X}_{i}, \hat{X}_{j}\right]=c_{i j}^{k} \hat{X}_{k}
$$

Each generator in the original basis can be written in terms of the new basis as follows:

$$
X_{i}=b_{i}^{l} \hat{X}_{l}, \quad i=1, \ldots, R,
$$

where $\operatorname{det}\left(b_{i}^{l}\right) \neq 0$. By substituting (2.7) into (2.5) and taking (2.6) into account, we find that the constants $b_{i}^{l}$ satisfy the nonlinear constraints

$$
c_{l m}^{n} b_{i}^{l} b_{j}^{m}=c_{i j}^{k} b_{k}^{n}
$$

(The identity $c_{j i}^{k}=-c_{i j}^{k}$ implies that it is sufficient to assume that $i<j$ in (2.8); we shall do this henceforth.) From (2.7), we obtain a system of $R(M+N)$ first-order PDEs for the $M+N$ unknown functions $(\hat{x}, \hat{u})$ :

$$
X_{i} \hat{x}^{s}=b_{i}^{l} \xi_{l}^{s}(\hat{x}, \hat{u}), \quad X_{i} \hat{u}^{\sigma}=b_{i}^{l} \eta_{l}^{\sigma}(\hat{x}, \hat{u}) .
$$

Every point symmetry of the given PDE necessarily satisfies (2.9) for some nonsingular matrix $B=\left(b_{i}^{l}\right)$. This system is generally easy to solve using the method of characteristics. Moreover, if $\operatorname{dim}(\mathscr{L})>M+N$, it is usually possible to solve (2.9) by eliminating the derivatives to obtain algebraic equations for $(\hat{x}, \hat{u})$.

The most straightforward way of determining the discrete symmetries is: first solve (2.9) for arbitrary $B$, then check which of the solutions satisfies the symmetry condition. The continuous symmetries should be factored out at any convenient stage to produce a list of discrete symmetries, no two of which are equivalent to one another under any symmetry in a one-parameter Lie group. 
If $\mathscr{L}$ is non-abelian, considerable effort may be saved by simplifying $B$ before solving (2.9). This is done by creating as many zero entries as possible, using the constraints (2.8) and the adjoint action of the continuous symmetries. The adjoint action of the one-parameter group generated by $X_{j}$ on the basis $\left\{X_{1}, \ldots, X_{R}\right\}$ is described by the $R \times R$ matrix

$$
A(j, \epsilon)=\exp \{\epsilon C(j)\}
$$

where

$$
(C(j))_{i}^{k}=c_{i j}^{k}
$$

The one-parameter Lie group generated by $X_{i}$ is equivalent, under the group generated by $X_{j}$, to the group generated by

$$
\tilde{X}_{i}=\operatorname{Ad}\left(e^{\epsilon X_{j}}\right) X_{i} \equiv(A(j, \epsilon))_{i}^{p} X_{p} .
$$

We can rewrite (2.7) as

$$
\tilde{X}_{i}=\tilde{b}_{i}^{l} \hat{X}_{l}
$$

where

$$
\tilde{b}_{i}^{l}=(A(j, \epsilon))_{i}^{p} b_{p}^{l} .
$$

Therefore (2.11) is equivalent, under the group generated by $X_{j}$, to

$$
X_{i}=\tilde{b}_{i}^{l} \hat{X}_{l} .
$$

The system (2.12) is similar to (2.7), the only difference being that the matrix $B$ in (2.7) has been replaced by $A(j, \epsilon) B$. In the same way, by considering the adjoint action of the group generated by $\hat{X}_{j}$ on the group generated by $\hat{X}_{i}$, we can replace $B$ by $B A(j, \epsilon)$. These equivalence transformations do not affect (2.8). By using each $A(j, \epsilon)$ in turn, one can usually factor out the continuous symmetries before solving (2.9).

In practice, it is most effective to write out the nonlinear constraints (2.8) and to solve as many as possible immediately. (I recommend the use of computer algebra if $\operatorname{dim}(\mathscr{L}) \geqslant 4$.) When no further simplification is possible, try to create an extra zero entry in $B$ by carrying out an equivalence transformation with a non-diagonal adjoint matrix $A(j, \epsilon)$. This simplifies the remaining nonlinear constraints, and so the procedure can be iterated. Finally, any diagonal adjoint matrices are used to rescale rows or columns of $B$. The matrices $A(j, \epsilon)$ may be used in any convenient order, but each one should be used exactly once. (For each $j$, the choice of a particular $\epsilon$ factors out the one-parameter Lie group generated by $X_{j}$.)

We shall consider equivalence under continuous symmetries with real-valued parameters; the inequivalent discrete symmetries may have a real or complex action on the variables $(x, u)$. Each real matrix $B$ that satisfies the nonlinear constraints (2.8) corresponds to a real automorphism of the Lie algebra. By factoring out equivalent matrices using the adjoint action of the Lie symmetries, we obtain the (factor) group of inequivalent real automorphisms. We then use the symmetry condition (2.3) to determine all discrete symmetries associated with each automorphism in this group. The number of such symmetries depends upon the PDE, whereas the number of inequivalent automorphisms is determined by the Lie algebra. Consequently there may be automorphisms that are not realised as symmetries, and some automorphisms may generate more than one symmetry. 


\section{A worked example: the spherical Burgers' equation}

To illustrate the method, consider the spherical Burgers' equation,

$$
u_{t}+\frac{u}{t}+u u_{x}=u_{x x}
$$

which has a three-dimensional Lie algebra of point symmetry generators [7]. These are spanned by

$$
X_{1}=-2 t \partial_{t}-x \partial_{x}+u \partial_{u}, \quad X_{2}=\ln t \partial_{x}+\frac{1}{t} \partial_{u}, \quad X_{3}=\partial_{x},
$$

whose nonzero commutators $\left[X_{i}, X_{j}\right], i<j$, are

$$
\left[X_{1}, X_{2}\right]=X_{2}-2 X_{3}, \quad\left[X_{1}, X_{3}\right]=X_{3} .
$$

Therefore the nonzero structure constants are

$$
c_{12}^{2}=1, \quad c_{12}^{3}=-2, \quad c_{13}^{3}=1, \quad c_{21}^{2}=-1, \quad c_{21}^{3}=2, \quad c_{31}^{3}=-1 .
$$

The matrices $C(j)$ are

$$
C(1)=\left[\begin{array}{rrr}
0 & 0 & 0 \\
0 & -1 & 2 \\
0 & 0 & -1
\end{array}\right], \quad C(2)=\left[\begin{array}{rrr}
0 & 1 & -2 \\
0 & 0 & 0 \\
0 & 0 & 0
\end{array}\right], \quad C(3)=\left[\begin{array}{lll}
0 & 0 & 1 \\
0 & 0 & 0 \\
0 & 0 & 0
\end{array}\right] .
$$

Exponentiating the matrices $\epsilon C(j)$, we obtain

$$
A(1, \epsilon)=\left[\begin{array}{ccc}
1 & 0 & 0 \\
0 & e^{-\epsilon} & 2 \epsilon e^{-\epsilon} \\
0 & 0 & e^{-\epsilon}
\end{array}\right], \quad A(2, \epsilon)=\left[\begin{array}{ccc}
1 & \epsilon & -2 \epsilon \\
0 & 1 & 0 \\
0 & 0 & 1
\end{array}\right], \quad A(3, \epsilon)=\left[\begin{array}{lll}
1 & 0 & \epsilon \\
0 & 1 & 0 \\
0 & 0 & 1
\end{array}\right]
$$

Now we write out the nonlinear constraints,

$$
c_{l m}^{n} b_{i}^{l} b_{j}^{m}=c_{i j}^{k} b_{k}^{n},
$$

for each $n$ in turn. It is usually best to begin with any $n$ for which $X_{n}$ is not in the subalgebra spanned by the commutators, because then the nonlinear terms in (3.2) vanish. For $n=1$, we obtain

$$
0=c_{i j}^{k} b_{k}^{1}
$$

Substituting $(i, j)=(1,3)$ into $(3.3)$, we find that $b_{3}^{1}=0$. Then $(i, j)=(1,2)$ yields $b_{2}^{1}=0$. The $3 \times 3$ matrix $B$ is nonsingular, so $b_{1}^{1} \neq 0$.

For $n=2$, the above results lead to to the constraints

$$
b_{1}^{1} b_{2}^{2}=b_{2}^{2}-2 b_{3}^{2}, \quad b_{1}^{1} b_{3}^{2}=b_{3}^{2} .
$$

Therefore

$$
b_{3}^{2}=0, \quad b_{3}^{3} \neq 0 .
$$

Finally, the remaining constraints (with $n=3$ ) are

$$
-2 b_{1}^{1} b_{2}^{2}+b_{1}^{1} b_{2}^{3}=b_{2}^{3}-2 b_{3}^{3}, \quad b_{1}^{1} b_{3}^{3}=b_{3}^{3} ;
$$

these yield the results

$$
b_{1}^{1}=1, \quad b_{2}^{2}=b_{3}^{3} \neq 0 .
$$


So far, we have been able to simplify $B$ to the following:

$$
B=\left[\begin{array}{ccc}
1 & b_{1}^{2} & b_{1}^{3} \\
0 & b_{2}^{2} & b_{2}^{3} \\
0 & 0 & b_{2}^{2}
\end{array}\right]
$$

Now we use the adjoint matrices $A(j, \epsilon)$ to simplify the off-diagonal elements. Note that

$$
B A(2, \epsilon)=\left[\begin{array}{ccc}
1 & b_{1}^{2}+\epsilon & b_{1}^{3}-2 \epsilon \\
0 & b_{2}^{2} & b_{2}^{3} \\
0 & 0 & b_{2}^{2}
\end{array}\right]
$$

so (by choosing $\epsilon=-b_{1}^{2}$ ) this equivalence transformation enables us to replace $b_{1}^{2}$ by zero. Similarly, post-multiplying $B$ by $A\left(3,-b_{1}^{3}\right)$ is equivalent to setting $b_{1}^{3}=0$. Finally, post-multiplying $B$ by $A\left(1,-b_{2}^{3} /\left(2 b_{2}^{2}\right)\right)$ gives $b_{2}^{3}=0$. In summary, we have factored out the Lie symmetries by using the adjoint action, and the inequivalent discrete symmetries are those solutions of (2.9), with

$$
B=\left[\begin{array}{lll}
1 & 0 & 0 \\
0 & b & 0 \\
0 & 0 & b
\end{array}\right], \quad b \neq 0
$$

that also satisfy the symmetry condition. (N.B. The above simplication of $B$ could equally well be achieved by premultiplying $B$ by the adjoint matrices.)

The system (2.9), with $B$ as above, amounts to

$$
\left[\begin{array}{ccc}
X_{1} \hat{t} & X_{1} \hat{x} & X_{1} \hat{u} \\
X_{2} \hat{t} & X_{2} \hat{x} & X_{2} \hat{u} \\
X_{3} \hat{t} & X_{3} \hat{x} & X_{3} \hat{u}
\end{array}\right]=B\left[\begin{array}{ccc}
-2 \hat{t} & -\hat{x} & \hat{u} \\
0 & \ln \hat{t} & 1 / \hat{t} \\
0 & 1 & 0
\end{array}\right]=\left[\begin{array}{ccc}
-2 \hat{t} & -\hat{x} & \hat{u} \\
0 & b \ln \hat{t} & b / \hat{t} \\
0 & b & 0
\end{array}\right] .
$$

This system of first-order PDEs has the general solution

$$
\hat{t}=c_{1} t, \quad \hat{x}=b\left(x+\left(\ln c_{1}\right) t u+c_{3} t^{1 / 2}\right), \quad \hat{u}=\frac{b}{c_{1}} u+c_{2} t^{-1 / 2},
$$

where each $c_{i}$ is an arbitrary constant, and $c_{1} \neq 0$. All that remains is to substitute (3.5) into the symmetry condition

$$
\hat{u}_{\hat{t}}+\frac{\hat{u}}{\hat{t}}+\hat{u} \hat{u}_{\hat{x}}=\hat{u}_{\hat{x} \hat{x}} \quad \text { when } \quad u_{t}+\frac{u}{t}+u u_{x}=u_{x x} .
$$

(For the sake of brevity, the details of this straightforward calculation are omitted.) It turns out that the symmetry condition imposes the further constraints

$$
b^{2}=c_{1}=1, \quad c_{2}=c_{3}=0 .
$$

Therefore, there are two classes of discrete symmetries, namely those that are equivalent to

$$
\Gamma_{1}:(t, x, u) \mapsto(t,-x,-u),
$$

and those that are equivalent to $\left(\Gamma_{1}\right)^{2}$, which is the identity. In other words, the factor group of inequivalent discrete symmetries is isomorphic to the cyclic group $\mathbb{Z}_{2}$, and is generated by $\Gamma_{1}$.

In this example, most automorphisms of the Lie algebra do not generate any discrete symmetries. The automorphisms for which $b^{2}=1$ each generate a single discrete symmetry. 


\section{Discrete symmetries of the Harry-Dym equation and Burgers' equation}

Generally speaking, the complexity of the calculations that are needed to determine the inequivalent automorphisms of a Lie algebra, $\mathscr{L}$, increases with $R=\operatorname{dim}(\mathscr{L})$. The system of nonlinear constraints $(2.8)$ yields up to $R^{2}(R-1) / 2$ separate equations. However, once the set of inequivalent matrices $B$ has been found, it is usually easy to solve the determining equations (2.9). In this section, we use the method described above to derive the inequivalent discrete symmetries of two well-known PDEs. (The details of the calculations that produce the set of inequivalent automorphisms are outlined in the Appendix.)

The Harry-Dym equation,

$$
u_{t}=u^{3} u_{x x x}
$$

has a five-dimensional Lie algebra $\mathscr{L}$ of point symmetry generators [3]. The basis

$$
\begin{gathered}
X_{1}=\partial_{x}, \quad X_{2}=x \partial_{x}+u \partial_{u}, \quad X_{3}=x^{2} \partial_{x}+2 x u \partial_{u}, \\
X_{4}=\partial_{t}, \quad X_{5}=t \partial_{t}-\frac{u}{3} \partial_{u},
\end{gathered}
$$

has the following non-zero structure constants $c_{i j}^{k}, i<j$ :

$$
c_{12}^{1}=1, \quad c_{13}^{2}=2, \quad c_{23}^{3}=1, \quad c_{45}^{4}=1 .
$$

After solving the constraints (2.8) and using the adjoint matrices to factor out the Lie symmetries (see Appendix), we find that either

$$
B=\operatorname{diag}\{\alpha, 1, \alpha, \beta, 1\}
$$

or

$$
B=\left[\begin{array}{ccccc}
0 & 0 & \alpha & 0 & 0 \\
0 & -1 & 0 & 0 & 0 \\
\alpha & 0 & 0 & 0 & 0 \\
0 & 0 & 0 & \beta & 0 \\
0 & 0 & 0 & 0 & 1
\end{array}\right]
$$

here $\alpha, \beta$ are each either 1 or -1 . Therefore, there are eight inequivalent real automorphisms of the Lie algebra. The general solution of (2.9) is

$$
\hat{t}=\beta t, \quad \hat{x}=\alpha x, \quad \hat{u}=c_{1} u,
$$

if $B$ is of the form (4.3); otherwise

$$
\hat{t}=\beta t, \quad \hat{x}=-\frac{\alpha}{x}, \quad \hat{u}=\frac{c_{1} u}{x^{2}} .
$$

By substituting each of these results into the symmetry condition, we find that the factor group of inequivalent real discrete symmetries is isomorphic to $\mathbb{Z}_{2} \otimes \mathbb{Z}_{2}$; it is generated by

$$
\begin{gathered}
\Gamma_{1}:(x, t, u) \mapsto(-x,-t, u), \\
\Gamma_{2}:(x, t, u) \mapsto\left(-\frac{1}{x}, t, \frac{u}{x^{2}}\right) .
\end{gathered}
$$

The group of inequivalent complex discrete symmetries is the direct product of the above 
group with the $\mathbb{Z}_{3}$ group generated by

$$
\Gamma_{3}:(x, t, u) \mapsto\left(x, t, e^{2 \pi i / 3} u\right) .
$$

The mappings $\Gamma_{1}$ and $\Gamma_{3}$ correspond to (4.3) with $\alpha=\beta=-1$ and $\alpha=\beta=1$ respectively, whereas $\Gamma_{2}$ corresponds to (4.4) with $\alpha=\beta=1$. In this example, the automorphisms with $\beta=-\alpha$ are not realized as point symmetries. Note that each of the discrete point symmetries generated by $\Gamma_{3}$ corresponds to the trivial automorphism $b_{i}^{l}=\delta_{i}^{l}$, so the inequivalent automorphisms with $\alpha=\beta$ each generate three complex symmetries.

So far, we have only seen examples in which the inequivalent discrete symmetries form either a cyclic group or a direct product of such groups. However, some PDEs have a more complicated structure. Burgers' equation,

$$
u_{t}+u u_{x}=u_{x x}
$$

has a five-dimensional Lie algebra of point symmetry generators [7], with a basis

$$
\begin{gathered}
X_{1}=\partial_{x}, \quad X_{2}=\partial_{t}, \quad X_{3}=x \partial_{x}+2 t \partial_{t}-u \partial_{u}, \\
X_{4}=2 t \partial_{x}+2 \partial_{u}, \quad X_{5}=4 t x \partial_{x}+4 t^{2} \partial_{t}+4(x-t u) \partial_{u} .
\end{gathered}
$$

After simplification of the matrix $B$ (as detailed in the Appendix), the possible inequivalent automorphisms are

$$
B=\operatorname{diag}\{\alpha a, \alpha, 1, a, \alpha\}
$$

and

$$
B=\left[\begin{array}{ccccc}
0 & 0 & 0 & -a & 0 \\
0 & 0 & 0 & 0 & \alpha \\
0 & 0 & -1 & 0 & 0 \\
\alpha a & 0 & 0 & 0 & 0 \\
0 & \alpha & 0 & 0 & 0
\end{array}\right]
$$

where $\alpha$ is either 1 or -1 , and $a$ is a real constant. By solving (2.9) and checking to see which of the solutions are symmetries, we obtain the following result. The inequivalent complex discrete symmetries of Burgers' equation form a group of order 8 that is isomorphic to the quaternion group $Q_{2}$; it is generated by

$$
\begin{gathered}
\Gamma_{1}:(x, t, u) \mapsto(-i x,-t, i u), \\
\Gamma_{2}:(x, t, u) \mapsto\left(\frac{x}{2 t},-\frac{1}{4 t}, 2(t u-x)\right) .
\end{gathered}
$$

(The inequivalent real discrete symmetries are generated by $\Gamma_{2}$; they form the cyclic group $\mathbb{Z}_{4}$.)

Rational symmetries, such as those generated by $\Gamma_{2}$ in the above example, typically correspond to a Weyl reflection of an sl(2) subalgebra. For Burgers' equation, the subalgebra spanned by $X_{2}, X_{3}$, and $X_{5}$ is isomorphic to $\mathfrak{s l}(2)$. The Harry-Dym equation (4.1) also has discrete rational symmetries associated with the $s \mathfrak{l}(2)$ subalgebra $\operatorname{Span}\left(X_{1}, X_{2}, X_{3}\right)$. 


\section{Nonlocal discrete symmetries}

The method can readily be extended to deal with other types of symmetry, provided that the infinitesimal generators form a Lie algebra. Consider the following system of equations from gas dynamics (see Ibragimov [7] for details):

$$
q_{s}-v_{y}=0, \quad v_{s}+p_{y}=0, \quad p_{s}+\frac{3 p}{q} v_{y}=0 .
$$

Note that the first equation of (5.1) implies the existence of a potential, $\phi$, such that

$$
q=\phi_{y}, \quad v=\phi_{s}
$$

The system (5.1) has an eight-dimensional Lie algebra, $\mathscr{L}$, of quasi-local symmetry generators:

$$
\begin{gathered}
X_{1}=\partial_{s}, \quad X_{2}=s \partial_{s}-p \partial_{p}+q \partial_{q}+\phi \partial_{\phi}, \\
X_{3}=\partial_{y}, \quad X_{4}=y \partial_{y}+p \partial_{p}-q \partial_{q}, \\
X_{5}=\partial_{v}+s \partial_{\phi}, \quad X_{6}=v \partial_{v}+p \partial_{p}+q \partial_{q}+\phi \partial_{\phi}, \\
X_{7}=\partial_{\phi}, \quad X_{8}=s^{2} \partial_{s}+(\phi-s v) \partial_{v}-3 s p \partial_{p}+s q \partial_{q}+s \phi \partial_{\phi} .
\end{gathered}
$$

The quasi-local generators are point symmetry generators for the extended system (5.1), (5.2). Therefore we can use the new method to determine the inequivalent discrete quasilocal symmetries of (5.1). For brevity, we state the main results without giving details of their derivation, which follows the pattern laid down in the previous examples.

Note that $X_{1}, \ldots, X_{6}$, with $\phi$ projected out, generate point symmetries of (5.1). The span of these generators is a Lie subalgebra that is isomorphic to the direct sum of three copies of the two-dimensional affine algebra $\mathfrak{a}(1)$. There are forty-eight inequivalent matrices $B$ associated with this subalgebra. Eight of these matrices are diagonal, each one corresponding to a local discrete symmetry of the system. The other 40 matrices, which involve nontrivial permutations of the a(1) subalgebras, do not generate symmetries of the system.

To find the discrete quasi-local symmetries of (5.1), first calculate the automorphisms of the eight-dimensional Lie algebra $\mathscr{L}$. Then use the symmetry condition for the extended system (5.1), (5.2), to obtain all inequivalent discrete symmetries by the usual method. These quasi-local discrete symmetries form a group of order 16, which is generated by

$$
\begin{gathered}
\Gamma_{1}:(s, y, v, p, q, \phi) \mapsto\left(-\frac{1}{s}, y, \phi-s v,-s^{3} p,-\frac{q}{s},-\frac{\phi}{s}\right), \\
\Gamma_{2}:(s, y, v, p, q, \phi) \mapsto(-s, y,-v, p, q, \phi), \\
\Gamma_{3}:(s, y, v, p, q, \phi) \mapsto(s,-y,-v, p, q,-\phi) .
\end{gathered}
$$

The eight inequivalent discrete local point symmetries are generated by $\Gamma_{1}^{2}, \Gamma_{2}$ and $\Gamma_{3}$. As in the previous examples, there is an $\mathfrak{s I}(2)$ subalgebra, which gives rise to the rational symmetries. Here the nonlocal generator $X_{8}$ is part of that subalgebra, and therefore the rational symmetries are nonlocal. 


\section{Conclusion}

The new method presented in this paper can deal with a wide range of symmetries, both local and nonlocal. Work is in progress on extending the method to auto-Bäcklund transformations and equivalence transformations.

\section{Appendix: Details of calculations}

Here we outline the steps that lead to the matrices $B$ for the Harry-Dym equation and for Burgers' equation. Once these matrices have been found, it is easy to recover the discrete symmetries associated with each matrix by solving the determining equations (2.9). In each case, the Lie algebra is five-dimensional, and the determining equations can be solved algebraically.

For a five-dimensional Lie algebra, there are up to 50 nonlinear constraints (2.8); we shall refer to this set of nonlinear constraints as NC. Clearly, it is advisable to use computer algebra to carry out these calculations. At any stage, there are two choices open to us. Either we can solve part of $\mathrm{NC}$ to obtain one or more of the $b_{i}^{l}$, or we may try to simplify $B$ by multiplying it by a previously-unused matrix $A(j, \epsilon)$ and choosing $\epsilon$ appropriately. Once either of these operations have been carried out, the set NC should be simplified. This process is iterated until no further constraints remain. To present the calculations in a concise form, we shall not write down the set NC. (The interested reader should use computer algebra to write down this set.) Instead, we list the conclusions in the order in which they are found. Except where indicated, these conclusions are drawn directly from NC (taking into account the information that we already have).

\section{Harry-Dym equation}

For the Harry-Dym equation, the structure constants (4.2) yield

$$
\begin{gathered}
A(1, \epsilon)=\left[\begin{array}{ccccc}
1 & 0 & 0 & 0 & 0 \\
-\epsilon & 1 & 0 & 0 & 0 \\
\epsilon^{2} & -2 \epsilon & 1 & 0 & 0 \\
0 & 0 & 0 & 1 & 0 \\
0 & 0 & 0 & 0 & 1
\end{array}\right], \quad A(2, \epsilon)=\left[\begin{array}{ccccc}
e^{\epsilon} & 0 & 0 & 0 & 0 \\
0 & 1 & 0 & 0 & 0 \\
0 & 0 & e^{-\epsilon} & 0 & 0 \\
0 & 0 & 0 & 1 & 0 \\
0 & 0 & 0 & 0 & 1
\end{array}\right], \\
A(3, \epsilon)=\left[\begin{array}{ccccc}
1 & 2 \epsilon & \epsilon^{2} & 0 & 0 \\
0 & 1 & \epsilon & 0 & 0 \\
0 & 0 & 1 & 0 & 0 \\
0 & 0 & 0 & 1 & 0 \\
0 & 0 & 0 & 0 & 1
\end{array}\right], \quad A(4, \epsilon)=\left[\begin{array}{ccccc}
1 & 0 & 0 & 0 & 0 \\
0 & 1 & 0 & 0 & 0 \\
0 & 0 & 1 & 0 & 0 \\
0 & 0 & 0 & 1 & 0 \\
0 & 0 & 0 & -\epsilon & 1
\end{array}\right], \\
A(5, \epsilon)=\left[\begin{array}{ccccc}
1 & 0 & 0 & 0 & 0 \\
0 & 1 & 0 & 0 & 0 \\
0 & 0 & 1 & 0 & 0 \\
0 & 0 & 0 & e^{\epsilon} & 0 \\
0 & 0 & 0 & 0 & 1
\end{array}\right]
\end{gathered}
$$


To begin with, $\mathrm{NC}$ includes the equations

$$
b_{1}^{5}=b_{2}^{5}=b_{3}^{5}=b_{4}^{5}=0 ;
$$

therefore $b_{5}^{5} \neq 0$ (because the matrix $B$ is nonsingular). This leads to

$$
b_{1}^{4}=b_{2}^{4}=b_{3}^{4}=0 ;
$$

consequently $b_{4}^{4} \neq 0$ and hence $b_{5}^{5}=1$. Now pre-multiply $B$ by $A\left(4, b_{5}^{4} / b_{4}^{4}\right)$ to replace $b_{5}^{4}$ by zero. At this stage, there are two cases that must be examined in turn.

Suppose that $b_{1}^{1} \neq 0$. Premultiply $B$ by $A\left(1, b_{2}^{1} / b_{1}^{1}\right)$ to replace $b_{2}^{1}$ by zero. Then NC yields the following results:

$$
\begin{gathered}
b_{2}^{2}=1 ; \quad b_{3}^{1}=b_{4}^{1}=b_{5}^{1}=0 ; \\
b_{3}^{2}=b_{4}^{2}=b_{5}^{2}=b_{4}^{3}=b_{5}^{3}=0 ; \quad b_{3}^{3}=1 / b_{1}^{1} .
\end{gathered}
$$

Pre-multiply $B$ by $A\left(3,-b_{1}^{2} / 2\right)$ to replace $b_{1}^{2}$ by zero. Then

$$
b_{1}^{3}=b_{2}^{3}=0 .
$$

So far we have used the non-diagonal adjoint matrices to simplify $B$ to the form

$$
B=\left[\begin{array}{ccccc}
b_{1}^{1} & 0 & 0 & 0 & 0 \\
0 & 1 & 0 & 0 & 0 \\
0 & 0 & 1 / b_{1}^{1} & 0 & 0 \\
0 & 0 & 0 & b_{4}^{4} & 0 \\
0 & 0 & 0 & 0 & 1
\end{array}\right]
$$

Pre-multiply $B$ by $A\left(2,-\ln \left|b_{1}^{1}\right|\right)$ to replace $b_{1}^{1}$ by $\alpha= \pm 1$. Pre-multiply $B$ by $A\left(5,-\ln \left|b_{4}^{4}\right|\right)$ to replace $b_{4}^{4}$ by $\beta= \pm 1$. This completes the derivation of (4.3).

The only remaining possibility is that $b_{1}^{1}=0$. In this case, NC leads to

$$
b_{2}^{1}=b_{1}^{2}=0 \text {. }
$$

Therefore $b_{1}^{3} \neq 0$, and hence

$$
b_{2}^{2}=-1 ; \quad b_{4}^{1}=b_{5}^{1}=b_{4}^{2}=b_{5}^{2}=b_{4}^{3}=b_{5}^{3}=0 ; \quad b_{3}^{1}=1 / b_{1}^{3} .
$$

Premultiply $B$ by $A\left(1, b_{2}^{3} / b_{1}^{3}\right)$ to replace $b_{2}^{3}$ by zero. Hence

$$
b_{3}^{2}=b_{3}^{3}=0 \text {. }
$$

So far we have reduced $B$ to

$$
B=\left[\begin{array}{ccccc}
0 & 0 & b_{1}^{3} & 0 & 0 \\
0 & -1 & 0 & 0 & 0 \\
1 / b_{1}^{3} & 0 & 0 & 0 & 0 \\
0 & 0 & 0 & b_{4}^{4} & 0 \\
0 & 0 & 0 & 0 & 1
\end{array}\right]
$$

The only non-diagonal adjoint matrix that we have not yet used is $A(3, \epsilon)$. When $B$ is multiplied by this matrix, new nonzero entries are produced if $\epsilon \neq 0$. Thus the simplest $B$ is obtained by setting $\epsilon=0$. Now premultiply $B$ by $A\left(2,-\ln \left|b_{1}^{3}\right|\right)$ to replace $b_{1}^{3}$ by $\alpha= \pm 1$, and by $A\left(5,-\ln \left|b_{4}^{4}\right|\right)$ to replace $b_{4}^{4}$ by $\beta= \pm 1$. This completes the derivation of (4.4). 


\section{Burgers' equation}

For Burgers' equation, the nonzero structure constants $c_{i j}^{k}, i<j$, corresponding to the basis (4.6) are

$$
c_{13}^{1}=1, \quad c_{15}^{4}=2, \quad c_{23}^{2}=2, \quad c_{24}^{1}=2, \quad c_{25}^{3}=4, \quad c_{34}^{4}=1, \quad c_{35}^{5}=2 .
$$

By exponentiating the matrices $C(j)$, we obtain

$$
\begin{gathered}
A(1, \epsilon)=\left[\begin{array}{ccccc}
1 & 0 & 0 & 0 & 0 \\
0 & 1 & 0 & 0 & 0 \\
-\epsilon & 0 & 1 & 0 & 0 \\
0 & 0 & 0 & 1 & 0 \\
0 & 0 & 0 & -2 \epsilon & 1
\end{array}\right], \quad A(2, \epsilon)=\left[\begin{array}{ccccc}
1 & 0 & 0 & 0 & 0 \\
0 & 1 & 0 & 0 & 0 \\
0 & -2 \epsilon & 1 & 0 & 0 \\
-2 \epsilon & 0 & 0 & 1 & 0 \\
0 & 4 \epsilon^{2} & -4 \epsilon & 0 & 1
\end{array}\right], \\
A(3, \epsilon)=\left[\begin{array}{cccccccc}
e^{\epsilon} & 0 & 0 & 0 & 0 \\
0 & e^{2 \epsilon} & 0 & 0 & 0 \\
0 & 0 & 1 & 0 & 0 \\
0 & 0 & 0 & e^{-\epsilon} & 0 \\
0 & 0 & 0 & 0 & e^{-2 \epsilon}
\end{array}\right], \quad A(4, \epsilon)=\left[\begin{array}{ccccc}
1 & 0 & 0 & 0 & 0 \\
2 \epsilon & 1 & 0 & 0 & 0 \\
0 & 0 & 1 & \epsilon & 0 \\
0 & 0 & 0 & 1 & 0 \\
0 & 0 & 0 & 0 & 1
\end{array}\right], \\
A(5, \epsilon)=\left[\begin{array}{ccccc}
1 & 0 & 0 & 2 \epsilon & 0 \\
0 & 1 & 4 \epsilon & 0 & 4 \epsilon^{2} \\
0 & 0 & 1 & 0 & 2 \epsilon \\
0 & 0 & 0 & 1 & 0 \\
0 & 0 & 0 & 0 & 1
\end{array}\right]
\end{gathered}
$$

At this stage, it is not immediately obvious how to solve any single equation in $\mathrm{NC}$, because the structure constants yield a system of constraints that is quite highly-coupled. (By constrast, the Lie algebra of the Harry-Dym equation is the direct sum of an $\mathfrak{s l}(2)$ subalgebra and an a(1) subalgebra, and some constraints are decoupled.) Note that $\operatorname{Span}\left(\mathrm{X}_{2}, \mathrm{X}_{3}, \mathrm{X}_{5}\right)$ is an $\mathfrak{s}(2)$ Lie subalgebra, as is $\operatorname{Span}\left(\mathrm{X}_{1}, \mathrm{X}_{2}, \mathrm{X}_{3}\right)$ in the Lie algebra of the Harry-Dym equation. Therefore, by analogy with the classification for the Harry-Dym equation, we treat the cases $b_{2}^{2} \neq 0$ and $b_{2}^{2}=0$ separately.

Suppose that $b_{2}^{2} \neq 0$. Premultiply $B$ by $A\left(2, b_{3}^{2} / 2 b_{2}^{2}\right)$ to replace $b_{3}^{2}$ by zero. Then NC yields

$$
\begin{gathered}
b_{3}^{3}=1 ; \quad b_{1}^{2}=b_{4}^{2}=b_{5}^{2}=b_{1}^{3}=b_{4}^{3}=b_{5}^{3}=0 ; \\
b_{1}^{5}=b_{4}^{5}=b_{4}^{1}=b_{5}^{1}=0 ; \quad b_{5}^{5}=1 / b_{2}^{2} .
\end{gathered}
$$

Therefore $b_{4}^{4} \neq 0$. Premultiply $B$ by $A\left(5,-b_{1}^{4} / 2 b_{4}^{4}\right)$ to replace $b_{1}^{4}$ by zero; as a result of this, we require that $b_{1}^{1} \neq 0$. Then

$$
b_{2}^{3}=b_{2}^{4}=b_{2}^{5}=b_{3}^{5}=0 .
$$

Pre-multiply $B$ by $A\left(1, b_{3}^{1} / b_{1}^{1}\right)$ to replace $b_{3}^{1}$ by zero. From NC,

$$
b_{5}^{4}=0 .
$$

Pre-multiply $B$ by $A\left(4,-b_{3}^{4} / b_{4}^{4}\right)$ to replace $b_{3}^{4}$ by zero. Therefore

$$
b_{2}^{1}=0 ; \quad b_{1}^{1}=b_{2}^{2} b_{4}^{4} .
$$


Finally, pre-multiply $B$ by $A\left(3,-\left(\ln \left|b_{2}^{2}\right|\right) / 2\right)$ to replace $B$ by (4.7).

To complete the classification, we now consider the case $b_{2}^{2}=0$. NC yields

$$
b_{3}^{2}=b_{2}^{3}=b_{1}^{2}=b_{1}^{3}=b_{4}^{2}=b_{4}^{3}=b_{1}^{5}=b_{1}^{1}=b_{4}^{5}=0 .
$$

Consequently $b_{5}^{2} \neq 0, b_{1}^{4} \neq 0$ and $b_{4}^{1} \neq 0$, and therefore

$$
b_{3}^{3}=-1 ; \quad b_{2}^{5}=1 / b_{5}^{2} ; \quad b_{2}^{1}=0 .
$$

Pre-multiply $B$ by $A\left(2, b_{4}^{4} / 2 b_{1}^{4}\right)$ to replace $b_{4}^{4}$ by zero. Then

$$
b_{3}^{5}=b_{5}^{3}=b_{5}^{5}=b_{5}^{4}=0 .
$$

Pre-multiply $B$ by $A\left(1, b_{3}^{4} / b_{1}^{4}\right)$ to replace $b_{3}^{4}$ by zero. Hence

$$
b_{5}^{1}=0 .
$$

Pre-multiply $B$ by $A\left(4,-b_{3}^{1} / b_{4}^{1}\right)$ to replace $b_{3}^{1}$ by zero. Thus

$$
b_{2}^{4}=0 ; \quad b_{4}^{1}=-b_{1}^{4} b_{5}^{2} .
$$

The matrix acquires new nonzero entries when it is multiplied by $A(5, \epsilon)$ if $\epsilon \neq 0$, so we choose $\epsilon=0$ to obtain the simplest possible $B$. Finally, premultiply $B$ by $A\left(3,\left(\ln \left|b_{5}^{2}\right|\right) / 2\right)$ to obtain (4.8).

\section{References}

[1] Bluman, G. W. \& Kumei, S. (1989) Symmetries and Differential Equations. Springer-Verlag.

[2] Gaeta, G. \& RodríGuez, M. A. (1996) Determining discrete symmetries of differential equations. Nuovo Cimento B, 111, 879-891.

[3] Hereman, W. (1996) Symbolic software for Lie symmetry analysis. In: N. H. Ibragimov (ed.), CRC Handbook of Lie Group Analysis of Differential Equations, vol. 3, pp. 367-413. CRC Press.

[4] Hydon, P. E. (1998) Discrete point symmetries of ordinary differential equations. Proc. Roy. Soc. Lond. A 454, 1961-1972.

[5] Hydon, P. E. (1998) How to find discrete contact symmetries. J. Nonlin. Math. Phys. 5, 405-416.

[6] Hydon, P. E. (2000) Symmetry Methods for Differential Equations. Cambridge University Press.

[7] Ibragimov, N. H. (1994) CRC Handbook of Lie Group Analysis of Differential Equations, vol. 1. CRC Press.

[8] Olver, P. J. (1993) Applications of Lie Groups to Differential Equations (2nd edn). SpringerVerlag.

[9] Reid, G. J., Wein, D. T. \& WitTKopf, A. D. (1993) A point symmetry group of a differential equation which cannot be found using infinitesimal methods. In: N. H. Ibragimov, M. Torrisi \& A. Valenti (eds.), Modern Group Analysis: Advanced analytical and computational methods in mathematical physics, pp. 311-316. Kluwer.

[10] Stephani, H. (1989) Differential Equations: Their solution using symmetries. Cambridge University Press. 